\title{
MONNIER116-5000 活塞调节阀的工作原理及常见故障分析
}

\section{Working Principle and Common Faults Analysis of MONNIER116-5000 Piston Regulating Valve 徐力 邱博 陈朝晖}

Li Xu Bo Qiu Zhaohui Chen

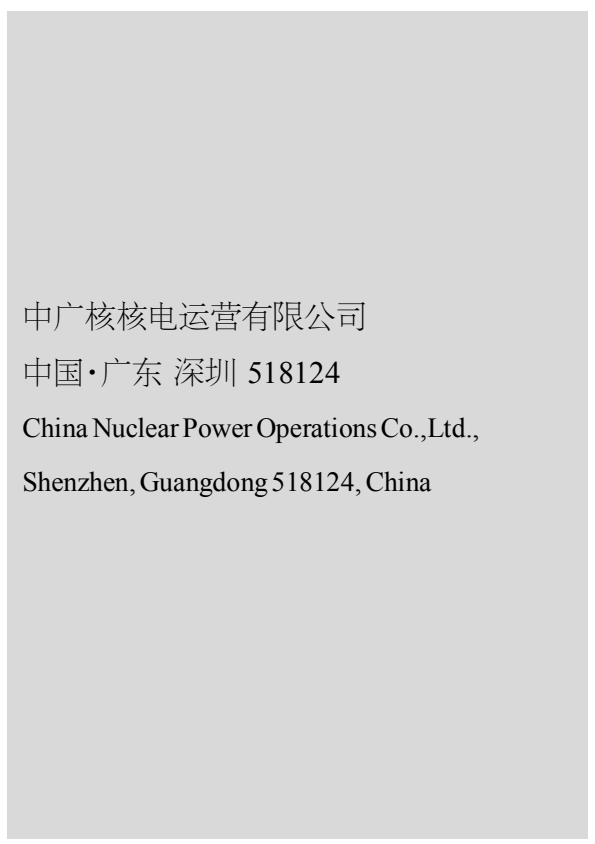

【摘 要】MONNIER116-5000 活塞调节阀常用于空气压力的整定。实际使用中,该型号调 节蔺经常发生下游气体压力剧烈波动、漏气、设备拒动等故障现象。进一步分析总结, 发现 该型号调节沎主要有下部活塞卡涩、润滑不良两种故障模式。论文通过对该型号调节伡结 构、工作原理、故障机理的分析, 可为后续的设备维护、故障处理提供重要参考, 也可为系 统调节阔的选型提供借鉴。

【Abstract】MONNIER116-5000 piston regulating valves are often used for setting air pressure. In actual use, the valve often produces the downstream gas pressure violent fluctuation, air leakage, equipment rejection and other failures. After further analysis and summary, it is found that the valve mainly has two failure modes: the lower piston jam and the poor lubrication. Through the analysis of the structure, working principle and fault mechanism of the model regulating valve, this paper can provide important reference for the subsequent equipment maintenance and fault treatment, and also provide reference for the selection of the system regulating valve.

【关键词】调节伡;卡涩; O-ring; 老化

【Keywords \regulating valve; jam; O-ring; aging

【DOI】10.36012/peti.v2i1.1306

\section{1 结构及工作原理}

\section{1 结构}

如图 1 所示: MONNIER116-5000 活塞调节阀(下文简称 调节阀), 主要由(1)紧固螺栓、(2)上部阀盖、(3)上部弹簧、(4)上 部活塞、(5)阀体、(6)下部活塞、(7)密封 O-ring、8下部弹簧、(9) 下部弹簧垫片、(10)下部阀盖、(11)调节手柄组成。

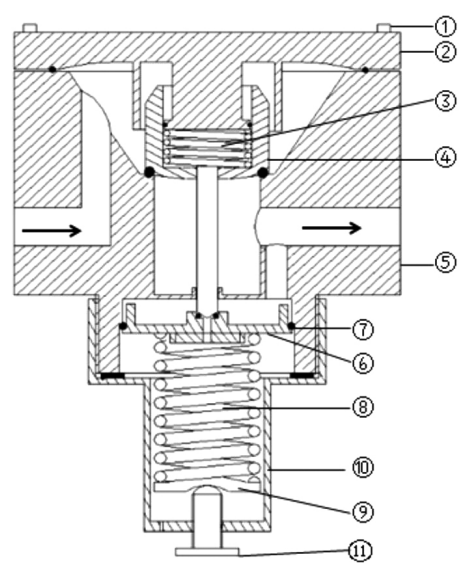

图 1 调节阀结构图

\section{2 工作原理}

调节阀主要用于空气压力的整定。某电厂厂用供气系统 空气压力约 $8 \mathrm{bar}^{[1]}$, 使用调节阀可将下游空气压力调整为 $0 \sim$ 8 bar 之间各个值。

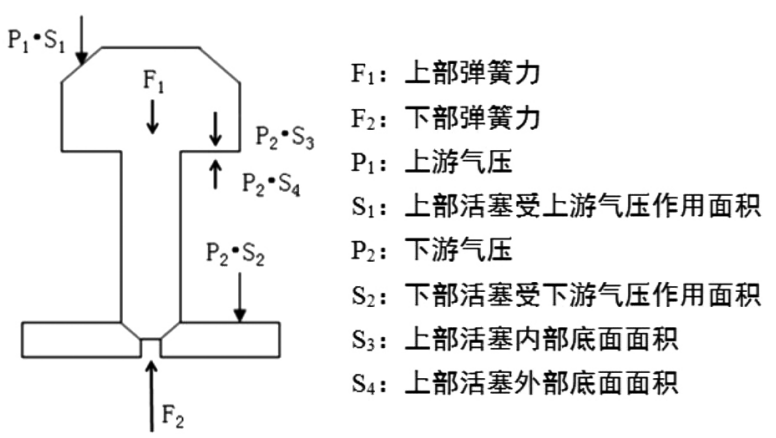

图 2 调节阀活塞受力示意图

如图 1 及图 2 所示。 $F_{1}$ 较小, 此处忽略不计。根据基础物 理知识 $F=P S$ ( $F$ 指作用力, $P$ 指气压, $S$ 指受压面积 $)$, 上游气 体在 $S_{1}$ 上的作用力为 $P_{1} S_{1}$ 。下游气体在 $S_{2}$ 上的作用力为 $P_{2} S_{2}$ 。 因上部活塞活塞头与活塞杆存在间隙, 所以下游气体能 进人上部活塞活塞头内部, 会产生向下的力 $P_{2} S_{3}$ 。因 $S_{3}$ 与 $S_{4}$ 
基本相等, 故 $P_{2} S_{3}$ 与 $P_{2} S_{4}$ 两力抵消, 此处忽略不计。所以, 当 调节戈达到平衡状态时, 有:

$$
P_{1} S_{1}+P_{2} S_{2}=F_{2}
$$

因 $P_{1} 、 S_{1} 、 S_{2}$ 恒定不变, 故 $P_{2}$ 与 $F_{2}$ 成直线线性相关。也就是 $F_{2}$ 增大或减小,那么 $P_{2}$ 也会随之增大或减小。 $F_{2}$ 大小与下部弹 簧压缩量有关。所以, 可通过调整下部弹簧的压缩量, 来调节下 游气压的大小。当下游气体被使用, $P_{2}$ 降低, $F_{2}$ 相应降低, 下部 弹簧压缩量变小, 上部活塞及下部活塞整体向上运动, 上部活 塞开启, 上游气体进入下游, $P_{2}$ 升高, 直至达到新的平衡。

\section{2 常见故障及原因分析}

根据维修经验, 调节阀主要有下部活塞卡涩、密封不良, 两种故障模式。

\section{1 下部活塞卡涩}

下部活塞卡涩是高发的故障模式, 主要原因有下部活塞 偏斜及润滑不良。上部活塞偏斜的原因主要有调节阀设计特 性、阀体及下部活塞材质因素、下部弹簧质量偏差、调节力不 能同轴线传递等。

(1)阀门特性。上部活塞、下部活塞并非一个整体, 下部活 塞可进行多角度及方向的运动, 这就是造成下部活塞偏斜的 先天因素。由图 3 可知, 下游气体反馈孔不是对称均匀分布 的, 所以在下部活塞频繁动作时, 下游气体对下部活塞的作用 力也无法均匀分布, 造成下部活塞偏斜,进而引发卡涩。

(2)阀体及下部活塞材质因素。阀体及下部活塞材质均为 铝镁合金, 铝含量大于 $98 \%$ 、镁含量约 $1 \%$, 其他元素不详。使 用中, 发现该材质极易磨损, 产生较严重的划痕, 如图 4 所示。 检修策略主要是打磨消除划痕, 打磨后下部活塞及对应阀体 腔室之间的间隙会变大, 进一步导致卡涩的频繁发生。

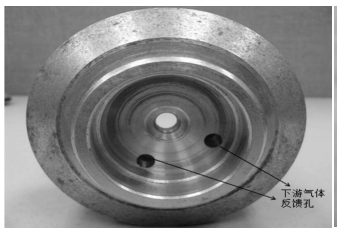

图 3 阀体下部腔室图

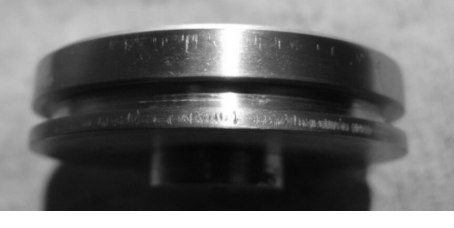

图 4 磨损划痕
(3)下部弹簧质量偏差。检查所有下部弹簧, 均存在偏斜情 况, 制造质量较差。因此, 下部弹簧不能沿坚直直线往复运 动, 这就会导致下部活塞往复运动时发生偏斜, 进而卡涩。

(4)调节力不能同轴线传递。下部弹簧与下部活塞径向配 合间隙约 $2 \mathrm{~mm}$, 间隙过大, 有相对位移空间。下部弹簧垫片是 锥帽型, 由两片组成, 不同心。下部活塞、下部弹簧、下部弹簧 座、调节手柄之间, 可进行多角度及方向的运动, 不能形成一 个坚直的轴线, 所以在调节过程中, 会造成下部活塞偏斜, 进 而引发卡涩。
(5)润滑不良。润滑不良有缺乏润滑和润滑脂涂抹过多两 种情况。调节阀长时间不维护,润滑脂干涸, 导致下部活塞与 腔室内壁摩擦力增大,引发卡涩。润滑脂涂抹过少, 下部活塞 与腔室内壁无法建立油膜, 导致卡涩。润滑脂涂抹太多,过量 的润滑脂挤压下部活塞的运动空间，增大下部活塞与腔室内 壁的黍连力, 导致卡涩。

\section{2 密封不良}

常见的密封不良主要有上部活塞与阀体之间密封不良、 下部活塞与戈体之间密封不良、上部活塞与下部活塞之间密 封不良。

(1)上部活塞与阀体之间密封不良。上部活塞 O-ring 老 化、失去弹性, 阀体与上部活塞 O-ring 对应密封面存在高点, 均会导致上部活塞与阀体之间密封不良，进而上游气体泄漏 进入下游, 导致下游气压升高。如图 5 所示, 上部活塞 O-ring 失去弹性有压痕, 无法像新 O-ring 一样恢复原状。

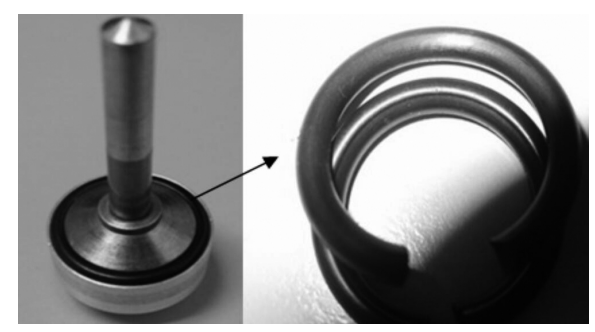

图 5 上部活塞新 0-ring 与失去弹性 0-ring 的对比

(2)下部活塞与阀体之间密封不良。下部活塞在静止和往 复运动的状态不停转换, 其外侧 O-ring 也在静密封和动密封 状态中不停转换。下部活塞往复运动时, 其 O-ring 与阀体内 壁摩擦扭转, 长时间使用后 O-ring 失去弹性, 无法补偿间隙, 导致下游气体泄漏。

(3)上部活塞与下部活塞之间密封不良。上部活塞活塞杆 锥面与下部活塞中心孔之间依靠 O-ring 进行密封。下部活塞 偏斜或上部活塞活塞杆晃度过大, 会导致两者接触不良, 导致 下游气体泄漏。该处 O-ring 的老化、失去弹性也会导致下游 气体泄漏。

\section{3 结论}

MONNIER116-5000 活塞调节阀, 在频繁动作中, 极易造 成下部活塞卡涩, 故不适用于频繁动作的系统, 例如, 不适合 为往复气动百进行直接供气。日常维护中, 应制定合理的维护 周期, 确保内部润滑有效; 及时更换密封 O-ring, 保证良好的 密封状态。

\section{参考文献}

[1]广东核电培训中心. $900 \mathrm{MW}$ 压水堆核电站系统与设备[M]. 北 京:原子能出版社,2007. 